\title{
EVISCERACIÓN INTESTINAL TRANSVAGINAL SECUNDARIA A TRAUMA GENITAL Y PERINEAL: PRESENTACIÓN DE UN CASO Y REVISIÓN DE LA LITERATURA
}

\section{Transvaginal intestinal evisceration after genital and perineal trauma: A case report and review of the liiterature}

Eder Alonso Ospino-Durán, $M D^{1}$; Carol Liliana Rodríguez-Amorocho, $\mathrm{MD}^{2}$; Luis Fernando Castaño-Molano, $M^{3}$; Carlos Julio García-Perlaza, $M^{3}$

Recibido: marzo 10/14 - Aceptado: septiembre 12/14

\section{RESUMEN}

Objetivo: presentar el manejo realizado a una mujer con evisceración intestinal transvaginal como consecuencia de un trauma genital y perineal; describir la etiología, el diagnóstico y el tratamiento de esta condición ginecológica mediante una revisión de la literatura.

Materiales y métodos: se presenta el caso de una mujer de 63 años de edad, con prolapso genital, quien sufre caída desde su propia altura que ocasiona trauma contundente y por aplastamiento a nivel genital y perineal; posteriormente se presenta disrupción de la pared vaginal y salida de asas intestinales, por lo que consultó a un hospital público de referencia ubicado en Bogotá, Colombia. Se realiza una búsqueda de la literatura en las bases de datos Medline vía PubMed, Science Direct y MD Consult, por medio de las palabras clave "vaginal",

1 Residente IV de Ginecología y Obstetricia, Universidad de La Sabana, Hospital Universitario de La Samaritana, Bogotá (Colombia). doctoreder04@hotmail.com

2 Residente III de Ginecología y Obstetricia, Universidad de La Sabana, Hospital Universitario de La Samaritana, Bogotá (Colombia).

3 Ginecólogo y obstetra. Profesor de Cirugía ginecológica, Hospital Universitario de La Samaritana, Bogotá (Colombia). “evisceration” y “trauma”. La búsqueda se limitó a artículos escritos en inglés y español.

Resultados: se encontraron 40 artículos. Se seleccionaron 16 estudios: un artículo descriptivo de serie de casos y el resto corresponden a reportes de caso. La mayoría de reportes de casos descritos corresponden a pacientes en etapa posmenopáusica con antecedente de cirugía pélvica ginecológica, aunque existen reportes en mujeres en edad fértil cuyo mecanismo causal es diferente. El diagnóstico se basa en la historia clínica y hallazgos al examen físico. Es considerada una urgencia quirúrgica. La vía del abordaje quirúrgico depende de los hallazgos al examen físico y de las condiciones médicas asociadas.

Conclusión: la evisceración transvaginal es una entidad poco frecuente, se considera una urgencia quirúrgica por las posibles complicaciones asociadas a la contaminación peritoneal o necrosis.

Palabras clave: prolapso de órgano pélvico, atrofia, intestino delgado.

\section{ABSTRACT}

Objective: To present the management of a female patients with transvaginal intestinal evisceration 
resulting from genital and perineal trauma, as well as to describe the aetiology, diagnosis and treatment of this gynaecological condition based on a review of the literature.

Materials and methods: Case presentation of a 63 year-old female patient with genital prolapse who fell from her own height and sustained blunt trauma and genital and perineal crush injury. Afterwards the patient presented disruption of the vaginal wall and evisceration of intestinal loops and was admitted at a referral public hospital in Bogotá, Colombia. A search was conducted in the PubMed, Science Direct and MD Consult databases using the key words "vaginal", "evisceration" and "trauma". The search was limited to papers written in English and Spanish.

Results: Overall, 40 articles were found. Of them, 16 studies were selected: one descriptive case series while the remaining were case reports. The majority of the case reports described referred to post-menopausal patients with a history of pelvic gynaecological surgery, although there were reports of women of childbearing age with a different causal mechanism. Diagnosis is based on the clinical history and physical examination findings. It is considered to require urgent surgery and the surgical approach depends on the physical findings and the associated medical conditions.

Conclusion: Transvaginal evisceration is a rare occurrence but it is considered to require urgent surgery because of potential complications associated with peritoneal contamination or necrosis.

Key words: Pelvic organ prolapse, atrophy, small intestine.

\section{INTRODUCCIÓN}

El primer caso de evisceración transvaginal reportado en la literatura fue realizado por Hyermaux en 1864 (1), definido como la extrusión del contenido intraperitoneal secundaria a una disrupción de las paredes de la vagina (2). Esta entidad es poco frecuente, con una incidencia de 0,032\% luego de una cirugía pélvica (3) y menos de $1 \%$ asociado a trauma perineal (4). La estructura más comúnmente identificada a través de la evisceración vaginal es el íleon; sin embargo, se ha reportado herniación del omento, apéndice y trompas uterinas, por lo que es una entidad que amerita una valoración y corrección quirúrgica urgente (5).

Se conoce que los antecedentes quirúrgicos ginecológicos, la atrofia genital, la pérdida de la laxitud de las estructuras de soporte de los órganos pélvicos y la disminución de la vascularización epitelial vaginal son factores predisponentes para que ocurra la disrupción y evisceración vaginal (6), dichos factores son generados por niveles bajos de estrógeno en la etapa senil y predisponen a la ulceración y disrupción de las paredes vaginales frente a un trauma (1, 7-9).

Debido a que la mayoría de casos clínicos reportados en la literatura describen mujeres con antecedentes quirúrgicos ginecológicos vía vaginal y abdominal, este caso adquiere importancia ya que ocurre secundario a trauma genital y perineal en ausencia de dichos antecedentes quirúrgicos. Además, no se conocen antecedentes de reportes bibliográficos de esta entidad en la población colombiana.

El objetivo de este artículo es describir la etiología, el diagnóstico y el manejo de la evisceración vaginal por medio de la presentación y el análisis de un caso clínico.

\section{PRESENTACIÓN DEL CASO}

Paciente de 63 años, multípara, índice de masa corporal 28,4 kg/m², con antecedente de diabetes mellitus, hipertensión arterial y prolapso genital, sin historia de cirugías previas, quien ingresa remitida en el mes de febrero del 2014 al Hospital Universitario de La Samaritana -institución pública de mediana y alta complejidad, ubicada en Bogotá, caracterizada por ser el centro de referencia para el departamento de Cundinamarca (Colombia), con atención predominante de pacientes de estrato socioeconómico bajo- por una caída desde su propia altura que ocasiona trauma contundente y 
aplastamiento a nivel genital y perineal, con posterior dolor, sensación de masa vaginal y sangrado escaso genital. Al ingreso no presenta otros síntomas asociados.

$\mathrm{Al}$ examen físico se evidencia prolapso genital (figura 1) POP-Q IVC (pelvic organ prolapse quantification system) (10) con cambios atróficos en mucosa vaginal asociados a ruptura del fondo de saco posterior y lateral derecho de $7 \mathrm{~cm}$ a través del cual protruyen 15 cm de intestino delgado sin signos de encarcelación ni isquemia. Al ingreso se realiza una reducción manual vía vaginal de las asas intestinales evisceradas y cobertura del sitio de continuidad a nivel vaginal con una bolsa estéril de líquidos endovenosos de polivinilcloruro o bolsa viaflex (figura 2), asociado a cubrimiento antibiótico con ampicilina sulbactam. En los estudios de laboratorio se documenta síndrome anémico (hemoglobina 8,9 mg/dl).

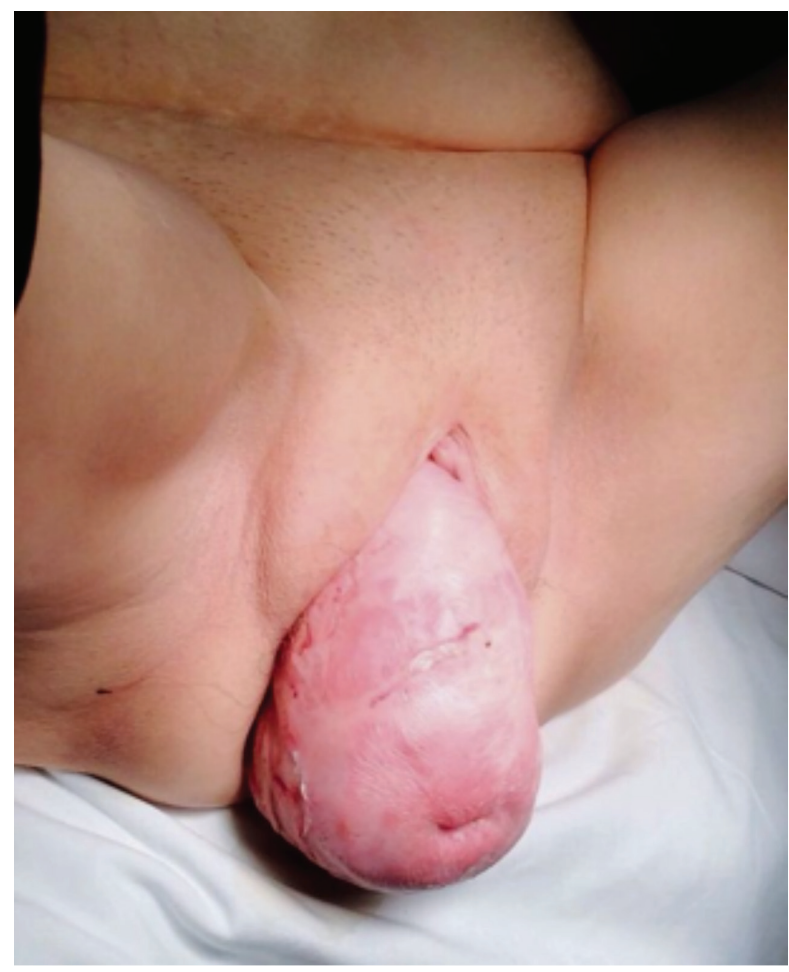

Figura 1. Prolapso genital POP-Q IVC, evidenciado en la paciente
Seis horas después del ingreso es llevada a corrección quirúrgica, con participación del servicio de ginecología, el cual realiza histerectomía vaginal + colporrafia anterior/posterior + corrección de enterocele y fijación bilateral de cúpula vaginal a ligamento sacroespinoso (figuras 3, 4, 5); el servicio de cirugía general realiza un lavado pélvico vía vaginal.

Durante la cirugía presenta descompensación hemodinámica y metabólica asociada al antecedente médico de diabetes y choque hipovolémico -teniendo en cuenta la hemoglobina preoperatoria, la pérdida sanguínea permisible en el acto quirúrgico era mínima-, con trastorno severo de la oxigenación, por lo que requiere transfusión de hemoderivados, ventilación mecánica invasiva y estancia en unidad de cuidado intensivo por cuatro días. Una semana posterior al ingreso, tras evolución clínica



Figura 2. Defecto de $7 \mathrm{~cm}$ a nivel de fondo de saco posterior y lateral derecho. Se observa bolsa estéril de polivinilcloruro mediante la cual se reducen asas intestinales evisceradas mientras es llevada a procedimiento quirúrgico 


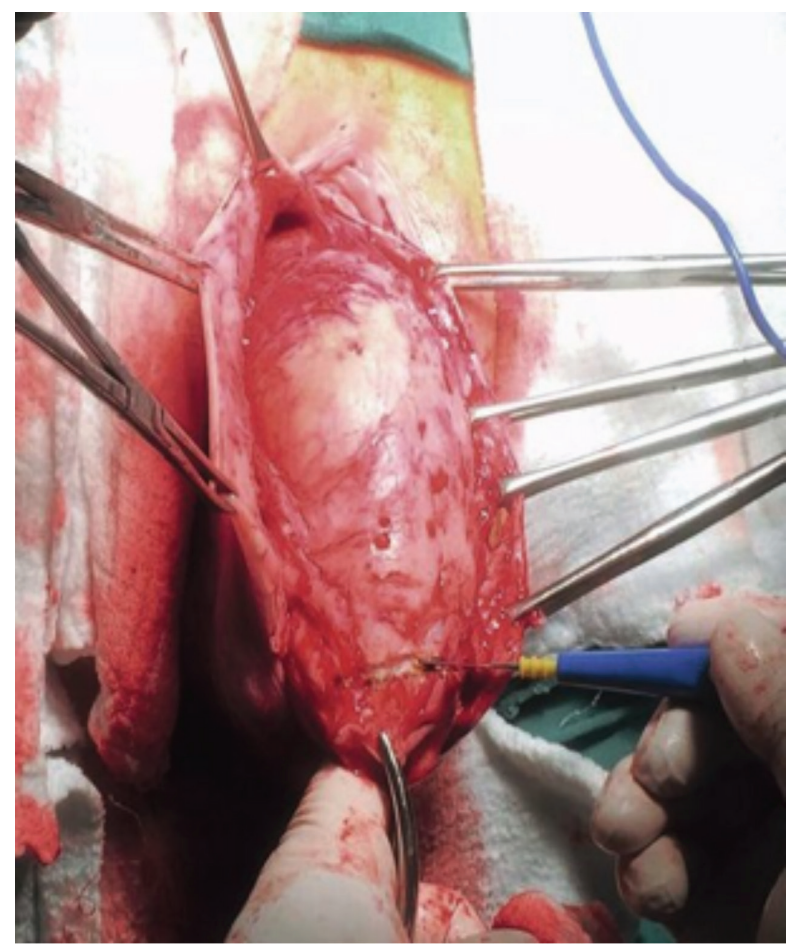

Figura 3. Disección de mucosa vaginal, fascia vesicovaginal y circuncisión cervical para lograr la extracción uterina

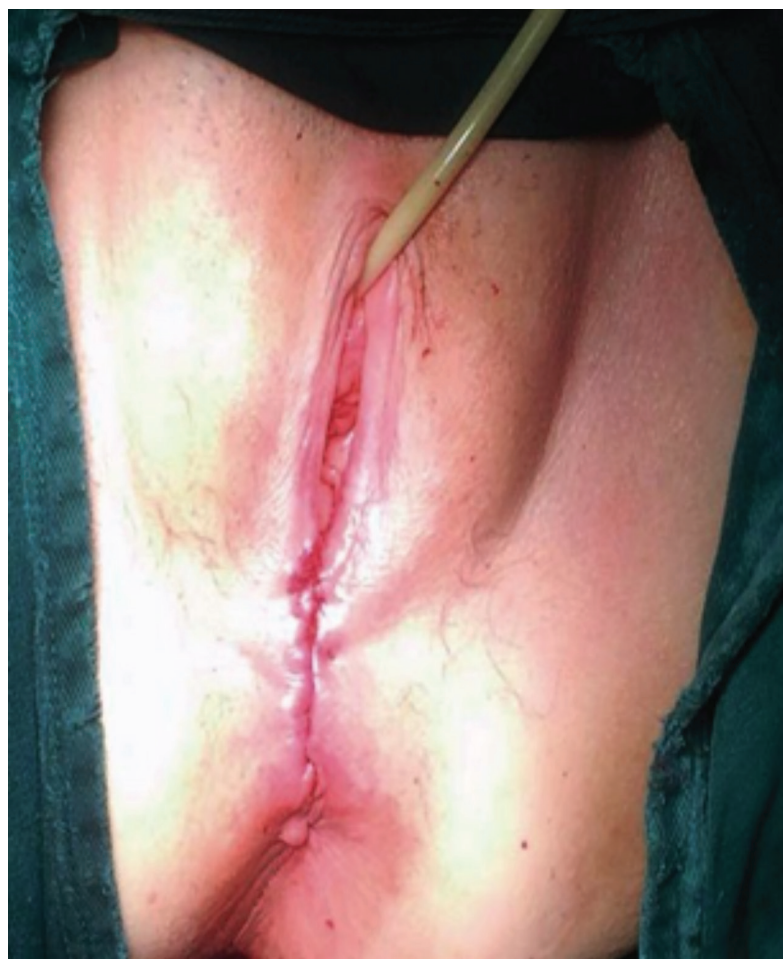

Figura 5. Aspecto físico de los genitales externos inmediatamente posterior al procedimiento quirúrgico



Figura 4. Lavado de cavidad pélvica vía vaginal con 2000 cc de solución salina, con retorno de líquido claro, una vez se logra la extracción uterina

satisfactoria, se decide dar el alta. Asiste a control médico ambulatorio donde se evidencia herida quirúrgica con adecuado proceso de cicatrización.

Aspectos éticos. Se solicitó autorización al paciente mediante un consentimiento informado escrito y al Hospital Universitario de La Samaritana, a través de su centro de investigación (CIHUS), para la publicación del caso clínico y el uso del archivo fotográfico. Se tomaron todas las precauciones para garantizar la confidencialidad del paciente.

\section{MATERIALES Y MÉTODOS}

Usando los términos "vaginal", "evisceration" y "trauma”, con "AND” como conector boleano, se realizó una búsqueda en las bases de datos Medline vía PubMed, Science Direct y MD Consult de artículos que hicieran referencia a la etiología, el diagnóstico y tratamiento de la evisceración vaginal. La búsqueda se limitó a documentos en inglés y español. 


\section{RESULTADOS}

Se encontraron 40 artículos, de los cuales se seleccionaron 16 estudios; entre los artículos escogidos hay uno descriptivo de serie de casos y el resto corresponde a reportes de caso.

Etiología. Usualmente se presenta en mujeres posmenopáusicas, aunque también han sido reportados casos de mujeres en edad fértil, siendo diferente la etiología o el mecanismo causal. En pacientes premenopáusicas se asocia a trauma durante el coito, asalto sexual o introducción vaginal de cuerpos extraños (1), mientras que en mujeres posmenopáusicas ocurre asociado al antecedente de cirugía pélvica vía vaginal, abdominal o laparoscópica $(1,8,11)$, por lo que la técnica quirúrgica, la infección del sitio operatorio, los hematomas y el inicio del coito en la fase posoperatoria juegan un papel muy importante en su desarrollo. Se han reportado casos por uso de pesario (12) y otros en ausencia de trauma, en donde el evento desencadenante es el aumento repentino de presión intraabdominal (por defecación, tos o el levantamiento de objetos pesados) (13-15).

En una serie de casos (12 pacientes) realizada por Mayo Clinic Foundation (3) con esta patología, el $75 \%$ tenía el antecedente de cirugía pélvica (33\% histerectomía vaginal y 42 \% histerectomía abdominal total), con un promedio de índice de masa corporal $27,9 \mathrm{~kg} / \mathrm{m}^{2}$; el sitio de ruptura más común fue a través del enterocele y fondo de saco posterior (67\% de los casos).

En esta ocasión, el caso reportado ocurre en una mujer posmenopáusica sin antecedente quirúrgico pélvico, con trauma directo genital y perineal como en otros reportes descritos $(2,11)$.

Diagnóstico. El diagnóstico de evisceración vaginal es clínico. Generalmente existe sensación de masa en canal vaginal, dolor abdominal bajo y sangrado genital de intensidad variable $(1,14)$; al examen físico se evidencian órganos abdominales o pélvicos que protruyen a través del introito vaginal, pero en algunos casos solamente se identifican al realizar una especuloscopia (4). Ayudas diagnósticas como la radiografía y la ecografía de abdomen no demuestran la extensión de la lesión (4).

El sitio más común de disrupción vaginal es el fondo de saco posterior (enterocele) y el órgano generalmente eviscerado es el intestino delgado $(1,13)$, situación evidenciada en el caso reportado.

Tratamiento. La evisceración vaginal es una condición médica asociada a complicaciones como peritonitis, sepsis y necrosis de asas intestinales (16), por lo que se considera una urgencia quirúrgica (14). Se han descrito cuatro fases $(1,3,8,9)$ para el tratamiento de la entidad: 1) reanimación hídrica y cubrimiento antibiótico; 2) reducción del contenido abdominal eviscerado previo lavado con solución estéril; 3) evaluación de viabilidad de estructuras evisceradas; 4) reparo quirúrgico inmediato.

La decisión del abordaje quirúrgico depende de los hallazgos al examen físico (2, 3), de las condiciones médicas asociadas y de la viabilidad de los tejidos prolapsados (17). La vía vaginal es preferida en aquellos pacientes con prolapso genital y viabilidad de estructuras evisceradas (14), condiciones cumplidas en el caso reportado, motivo por el cual fue la escogida para el tratamiento. La vía abdominal es usada cuando existen signos de isquemia intestinal o cuando la reposición de las estructuras prolapsadas es técnicamente difícil debido al compromiso del retorno venoso que genera edema (14). La vía laparoscópica, aunque es poco usada, tiene la ventaja teórica de que permite la exploración de la cavidad abdominal completa con menor riesgo quirúrgico y pronta recuperación (2).

En cuanto a los defectos del piso pélvico asociados (compartimento anterior, apical y posterior) existen dos momentos para su corrección. Se pueden reparar durante el mismo acto quirúrgico, como se realizó en este caso clínico, o se programa un segundo tiempo operatorio (1) con el fin de disminuir el riesgo de infección y mejorar la calidad de los tejidos mediante el uso de estrógeno vaginal. 


\section{CONCLUSIONES}

La evisceración transvaginal es una entidad poco frecuente. La mayoría de reportes de casos descritos en la literatura mundial corresponden a pacientes en etapa posmenopáusica con antecedente de cirugía pélvica ginecológica, aunque existen reportes en mujeres en edad fértil cuyo mecanismo causal es diferente. El diagnóstico se basa en la historia clínica y en hallazgos al examen físico. Es considerada una urgencia quirúrgica debido a que es una condición potencialmente mórbida. La vía del abordaje quirúrgico depende de los hallazgos al examen físico y condiciones médicas asociadas.

\section{AGRADECIMIENTOS}

A todo el personal médico y paramédico del Hospital Universitario de La Samaritana, Bogotá (Colombia), que participó en la atención de la paciente presentada en este artículo.

\section{REFERENCIAS}

1. Quiróz C, Martínez J, Rojano M, Beristain J, Moreno M. Evisceración vaginal. Informe de un caso y revisión de la bibliografía. Ginecol Obstet Mex. 2013;81: 349-52.

2. Bozkurt N, Korucuoglu U, Bakirci Y, Yilmaz U, Sakrak O, Guner H. Vaginal evisceration after trauma unrelated to previous pelvic surgery. Arch Gynecol Obstet. 2009;279:595-7.

3. Croak J, Gebhart J, Klingele C, Schroeder G, Lee R, Podratz K. Characteristics of patients with vaginal rupture and evisceration. Obstet Gynecol. 2004;103:572-6.

4. Fernando U, Wijesundera N, Goonesinghe S, Jayasooriya D. An injury resulting in vaginal evisceration. Ceylon Med J. 2010;55:124-5.

5. Ginsberg D, Rovner E, Raz S. Vaginal evisceration. Urology. 1998;51:128-9.
6. Ferrera P, Thibodeau L. Vaginal evisceration. The Journal of Emergency Medicine. 1999;17:665-7.

7. De Iaco P, Ceccaroni M, Alboni C, Roset B, Sansovini M, D'Alesandro L, et al. Transvaginal evisceration after hysterectomy: Is vaginal cuff closure associated with a reduced risk? European Journal Obstetrics \& Gynecology and Reproductive Biology. 2006;125: 134-8.

8. Nars A, Tormey S, Aziz M, Lane B. Vaginal herniation: case report and review of the literature. Am J Obstet Gynecol. 2005;193:95-7.

9. Kowalski L, Seski J, Timmins P, Kanbour A, Kunschner A, Kanbour-Shakir A. Vaginal evisceration: presentation and management in postmenopausal women. J Am Coll Surg. 1996;183:225-9.

10. Persu C, Chapple CR, Cauni V, Gutue S, Geavlete P. Pelvic Organ Prolapse Quantification System (POP-Q) - a new era in pelvic prolapse staging. J Med Life. 2011;4:75-81.

11. Austin J, Cooksey C, Minikel L, Zaritsky E. Postcoital vaginal rupture in a young woman with no prior pelvic surgery. J Sex Med. 2013;10:2121-4.

12. Rubin R, Jones K, Harmanli O. Vaginal evisceration during pessary fitting and treatment with immediate colpocleisis. Obstet Gynecol. 2010;116(Suppl 2): 496-8.

13. Rollinson D, Brodman M, Friedman F, Sperling R. Transvaginal small-bowel evisceration: a case report. Mt Sinai J Med. 1995;62:235-8.

14. Choo Y, Lindenauer S. Vaginal evisceration. Int J Gynaecol Obstet. 1981;19:313-7.

15. Friedel W, Kaiser I. Vaginal evisceration. Obstet Gynecol. 1975;45:315-9.

16. Nopdonrattakoon L. Vaginal vault rupture with evisceration of small intestine during coitus. J Med Assoc Thai. 2010;93:245-7.

17. Hall B, Phelan J, Pruyn S, Gallup D. Vaginal evisceration during coitus: a case review. Am J Obstet Gynecol. 1978;131:115-6. 